\title{
Effort toward comprehension: Elaboration or "aha!"?
}

\author{
PAMELA M. AUBLE, JEFFERY J. FRANKS, and SALVATORE A. SORACI, JR. \\ Vanderbilt University, Nashville, Tennessee 37240
}

\begin{abstract}
Auble and Franks (1978) found that a process termed "effort toward comprehension" was important in facilitating recall of sentences. Four experiments were conducted to further elucidate the nature of this process. Two hypotheses were considered: (1) Effort toward comprehension involves greater elaboration or deeper processing of the sentence; (2) effort toward comprehension can be viewed as an "aha" experience (i.e., a state of noncomprehension followed by comprehension of the sentence). Results indicated that recall was significantly greater for subjects in conditions producing "aha" reactions. No support was found for the elaboration interpretation of effort toward comprehension.
\end{abstract}

Auble and Franks (1978) present findings that indicate that a process termed "effort toward comprehension" can facilitate later recall. Using generally incomprehensible sentences and appropriate cues that render the sentences comprehensible, Auble and Franks (Experiment 2) found that presentation of a sentence followed by an appropriate cue facilitated recall relative to a condition in which the cue was meaningfully embedded in the sentence. This effect was attributed to processes involving differential degrees of effort toward comprehension. Further, in the former condition, in which the sentence and cue were presented separately, it was discovered that extra time during acquisition enhanced later recall, although extra time was not effective in the latter condition (Experiments 1 and 3). Auble and Franks (Experiment 4) also found that eventual comprehension of the sentence was necessary for the process of effort toward comprehension to be effective in facilitating recall. Given these initial demonstrations, the purpose of the present studies is to more closely examine the nature of the processes involved in effort toward comprehension. Two possible mechanisms that could underly this process will be considered.

The first possible mechanism will be termed the elaboration hypothesis. In this view, a greater amount of effort toward comprehension might be seen as related to or involving elaboration or deeper processing of the sentence (Craik \& Lockhart, 1972; Craik \& Tulving, 1975). Auble and Franks (1978, Experiments 1 and 2) discussed both elaborative processes occurring between the sentence and cue and elaborative processes occurring after the entire sentence-cue combination. Although these two potential types of elaborative processes could

This research was supported in part by National Science Foundation Grant BNS 77-07248, awarded to Jeffery Franks and John Bransford. The authors wish to express their thanks to Denise Wunderlich for her help in conducting and analyzing these experiments. not be distinguished on the basis of their results, it was found that longer time intervals were effective in promoting recall relative to conditions that received no time. This finding suggests that the underlying processes may involve some type of elaboration. That is, when a sentence and a cue are presented separately, subjects elaborate the sentence and its relation to the cue. This may be viewed as a process of activating concepts related to the words and/or subclauses of the difficult-tocomprehend sentences. These elaborated conceptual structures would be represented in the memory trace for the original sentence, and this elaborated representation would facilitate retrieval. Longer time intervals would allow greater degrees of such elaboration.

The second possible hypothesis posits that higher recall rates are related to subjects being momentarily in a state involving lack of comprehension and that this state is followed by understanding of the stimulus. This possibility, the "aha" hypothesis, posits that this "aha" response itself makes the sentence more memorable. Instead of attributing enhanced recall to elaboration of related conceptual structures, the "aha" hypothesis posits the locus of the effect to be related to the movement from a state of noncomprehension to a state of comprehension. This process may be characterized as a sequence of two encoded representations. The initial representation would encode the nonunderstood sentence without appropriate contextual reference. The following encoding, occurring with presentation of the cue, would re-represent the sentence, this time with appropriate contextual reference (derived from the cue) integrated into the initial representation. The precise mechanism by which this "aha" experience would lead to enhanced recall is not exactly clear, but some possibilities present themselves. One possibility is a form of elaboration processes. In particular, it may be that each of the two stages of representation in the "aha" process leaves a memory trace. This doubling or redundancy of memory traces could facilitate retrieval. A second 
possibility is that the "aha" process (the momentary state of noncomprehension followed by comprehension) itself is a distinctive informational state, and this distinctive information is represented with the encoded trace of the sentence. Against a background of everyday experience with sentences that are generally readily understood, the representation of the "aha" experience may form a distinctive retrieval cue for these relatively unusual, difficult-to-comprehend sentences. Although such accounts of mechanisms behind the elaboration and "aha" hypotheses are of interest, the primary concern of the present work is to assess the relative effectiveness of the elaboration and "aha" accounts as two general classes of explanations for the Auble and Franks' (1978) findings. A secondary purpose will be to shed some light on possible more precise mechanisms.

The basic contrast between the elaboration and "aha" hypotheses concerns whether enhanced recall is to be attributed (1) to elaboration of related concepts (while thinking about the sentence and cue) or (2) to a state of noncomprehension followed by comprehension involving an integrated representation of the sentence and cue information (an "aha" experience). Of course, it is possible that both processes are operative and that both contributed to the original findings of Auble and Franks (1978). However, these alternative accounts cannot be differentiated in those experiments. The findings that conditions in which the sentence is followed by the cue lead to enhanced recall relative to embedded cue conditions could be interpreted as support for the "aha" hypothesis. However, as discussed above, it may be that the effectiveness of the sentence-cue separation for recall is attributable to enhanced elaboration of related concepts rather than to the "aha" experience itself. Likewise, the original finding that recall is enhanced with longer time intervals may be seen as support for elaborative processes, but it could also be interpreted as being due to an "aha" process in which the integration of sentence and cue takes time to develop.

The present series of studies attempts to examine the extent to which either or both elaboration and "aha" processes are operative in experimental situations such as those of Auble and Franks (1978). In the present Experiment 1, the amount of elaborative processing involved in comprehending a sentence and cue and the occurrence of an "aha" experience were manipulated. Two types of sentences were used to vary the amount of elaborative processing involved in comprehension of a sentence. One type was similar to the sentences used by Auble and Franks. These sentences were generally incomprehensible without an appropriate cue ("difficult" sentences). An example of this type of sentence is "The haystack was important because the cloth ripped," with the cue, "parachute." The second group of sentences were termed "easy" sentences because they are relatively easy to understand before the appropriate cue is presented. For instance, a sentence such as "The office was cool because the windows were closed," is generally easily comprehended prior to presentation of the appropriate cue, "air conditioned." It was expected that the difficulty in comprehending the difficult sentences would lead subjects to think more about (i.e., elaborate more concepts related to) these sentences in comparison with the easy sentences. This increased elaboration should enhance recall.

In order to manipulate the potential occurrence of an "aha" reaction, both easy and difficult sentences were presented in two ways. Either the cue followed the sentence after a 5-sec delay (difficult-postcue, easypostcue), or the cue was meaningfully embedded in the sentence, making all sentences relatively normal and easily understandable (difficult-within, easy-within). An example sentence in the latter condition is "The airconditioned office was cool because the windows were closed." For both the easy-postcue and difficult-postcue conditions, it was expected that subjects would at least momentarily experience noncomprehension of the sentences and that this state would be followed by comprehension. That is, although the easy sentences are relatively easily understood without the cue, the processing of an easy-postcue sentence would at least momentarily still involve encoding an initial representation of the sentence as presented without the appropriate contextual reference. The initial representation would then be followed by a second encoding with the contextual reference integrated into the representation. (In the case of the easy-postcue sentences, the contextual referencing is usually provided from the subjects' background knowledge before the cue is presented.) The "aha" experience is not expected to occur for sentences in the relatively normal "within" conditions. In the within conditions, it was expected that the processing of both nominally difficult and easy sentences would be comparable and would involve immediately encoding a representation of the sentence including the appropriate contextual reference.

Thus, if the elaborative hypothesis holds, then recall of sentences in the difficult-postcue condition should be higher than recall of sentences in the easy-postcue condition. If the occurrence of an "aha" experience is importantly related to facilitated recall, then recall of both the postcue conditions should be higher than recall of the within conditions. Since, in the within conditions, both the difficult and easy sentences are actually relatively normal sentences that are easily comprehensible, it is expected that neither the elaborative nor the "aha" factors will be vitally involved in comprehending these sentences. Thus, all sentences in the within conditions should be equally well recalled.

\section{EXPERIMENT 1}

\section{Method}

Subjects. The subjects were 20 volunteers from the introductory psychology course at Vanderbilt University. They received course credit for their participation in the experiment.

Materials and Procedure. A set of 62 sentences, both easy and difficult, was constructed. Some of these sentences had been used previously (Auble \& Franks, 1978; Bransford \& McCarrell, 
1974; Johnson, Doll, Bransford, \& Lapinski, 1974); the rest were generated by the authors. These sentences were rated by an independent group of 19 introductory psychology students as to whether the sentences were understandable in isolation (i.e., without appropriate cues) and whether the sentences could be understood given the appropriate cues. Subjects were also given the opportunity to attempt to generate alternative contexts in which the sentences could be understood. (Sentences that either had more than one good contextual setting enabling comprehension or could not be understood in the presence of the cue were eliminated.) The 20 sentences that subjects reported understanding most of ten in isolation (mean $=88 \%, \mathrm{SD}=10.3$ ) were chosen to form a pool of easy sentences, and the 20 sentences that were reported as being understood without the cue least often (mean $=12 \%, S D=10.9)$ were chosen to form a pool of difficult sentences. All experiments reported herein used sentences from this 40 -item pool. Lists of both easy and difficult sentences with appropriate cues are provided in Appendix A. From this pool, a list was constructed of the 14 easy sentences that were understood without the cue most often (mean $=94 \%, \mathrm{SD}=5.3$ ) and of the 14 difficult sentences that were understood in isolation least often (mean $=6 \%$, $\mathrm{SD}=7.2$ ). This list of 28 sentences was used in Experiment 1 .

During acquisition, the 20 subjects participating in Experiment 1 were read this list of sentences in an intentional learning task. There were four types of sentences in the list. The list was composed half of easy sentences and half of difficult sentences. Half of both the easy and difficult sentences were presented with their appropriate cues $5 \mathrm{sec}$ after presentation of the sentence (easy-postcue, difficult-postcue). The remaining easy and difficult sentences were presented with the cue meaningfully embedded in the sentence and a 5-sec delay after the sentence (easy-within, difficult-within). These four types of sentences were randomly intermixed during presentation. Two acquisition lists were formed, and the postcue and within conditions were counterbalanced such that, across lists, each sentence appeared in each of the two conditions.

After the presentation of each sentence-cue combination and the 5-sec delay, the experimenter said "Mark," and the subjects rated whether they understood the sentence, did not understand it, or were unsure whether they understood the sentence or not. The subjects were given 2 sec to rate each sentence before the next sentence was presented. Immediately after all of the sentences were read, the experimenter instructed the subjects to recall as many of the sentences and their cues as possible. Subjects were given $9 \mathrm{~min}$ for free recall; partial recall was encouraged.

\section{Results and Discussion}

The understanding measure was scored, and the number of "understood," "not sure," and "did not understand" responses were computed. It was found that the sentences were generally well understood, although it appeared that easy sentences were understood slightly more often than difficult sentences. The comprehension rate ("understood" response) was $78 \%$ in the difficult-within condition, $80 \%$ in the difficult-postcue condition, $88 \%$ in the easy-within condition, and $99 \%$ in the easy-postcue condition.

Recall results were scored in terms of the number of sentences or sentence paraphrases recalled. The first sentence in the acquisition list and the last two sentences in the list were not included in the scoring to reduce primacy and recency effects. Recall was highest in the two postcue conditions (easy-postcue, mean $=29 \%$, $\mathrm{SD}=18.5 ;$ difficult-postcue, mean $=30 \%, \mathrm{SD}=22.6$ ) and relatively low in the two conditions in which the cue was meaningfully embedded in the sentence (easy-within, mean $=19 \%, \mathrm{SD}=19.7$; difficult-within, mean $=18 \%$, $\mathrm{SD}=15.6$ ). The data were analyzed by a 2 by 2 by 2 mixed design analysis of variance for both subjects and sentences. The three factors were the counterbalanced lists, the easy-difficult variable, and the cued-within variable. A significant main effect for the cued-within factor was obtained $\left[F_{1}(1,19)=6.21, p<.025\right.$; $\left.F_{2}(1,20)=7.88, p<.01\right]$. The effect due to the easydifficult factor was not significant for either analysis $\left(F_{1}<1, F_{2}<1\right)$, nor were any of the other main effects or interactions significant. The min $\mathrm{F}^{\prime}$ statistic (Clark, 1973) was marginally significant $\left[\min F^{\prime}(1,54)=3.55\right.$, $\mathrm{p}<.07]$. Because of the conservative nature of this test (Forster \& Dickinson, 1976) and of the fact that it is the minimum value for the true $F^{\prime}$, we feel justified in accepting this statistic as evidence for the generality of our results over sentences as well as subjects.

These results provide support for the notion that an "aha" experience is important in facilitating later recall. Recall was higher in the conditions in which "aha" reactions occurred (easy-postcue, difficult-postcue) relative to the easy-within and difficult-within conditions, in which such experiences were not present. On the other hand, the hypothesis that the amount of elaboration involved in comprehending the sentence is importantly related to later recall was not substantiated. Recall rates for the easy-postcue and difficult-postcue conditions were nearly identical, suggesting that the greater degree of elaboration of related conceptual structures presumably occurring in the difficult-postcue condition was not influential in facilitating later recall. As expected, recall rates in the easy-within and difficultwithin conditions were approximately equal, indicating that, comparatively, neither the "aha" factor nor the elaborative factor was operative for these relatively normal easily understood sentences.

Results from the present experiment thus suggest that facilitated recall is related to experiencing an "aha" reaction and that greater elaboration did not enhance recall. However, further consideration of the material employed in Experiment 1 suggested that recall in the easy-postcue condition could have been artifactually inflated, and any effects attributable to elaborative processing may have been obscured. Note that when easy sentences are used, results from the aforementioned norming data indicate that subjects comprehend the meaning of the sentence prior to presentation of the cue about $95 \%$ of the time. Thus, in the easy-postcue condition, presentation of the cue usually serves merely to reinforce the understood meaning of the sentence and could be regarded in this sense as a repetition of information that has already been obtained. In contrast, sentences in the difficultpostcue condition are generally not understood prior to the cue. The cue in these cases provides new information, enabling comprehension of the sentence. Thus, it may be that the explicit repetition of previously implicitly obtained information in the easy-postcue 
condition could have inflated later recall rates relative to the difficult-postcue condition. This might obscure any differences attributable to greater elaboration in the difficult-postcue condition. Thus, control of this factor may result in evidence supporting the notion that the amount of elaboration put forth in attempting to understand the sentence is an important factor in later recall.

To investigate this possibility, a second experiment was conducted, using easy and difficult materials as stimuli. The sentences were either followed by their appropriate cues as in Experiment 1 (easy-appropriate, difficult-appropriate) or followed by cues that were unrelated to the sentence (easy-inappropriate, difficultinappropriate). An example of the second condition would be a sentence such as "The office was cool because the windows were closed," followed by the cue, "covered wagons." For the easy-inappropriate condition, the cue now would not provide repetition of already known information. If repetition of information was an important factor in the results obtained for Experiment 1 , it would be expected that recall in the easy. appropriate condition would be higher than in the easy-inappropriate condition. This pattern of results could support the interpretation that the amount of elaborative processing occurring in the effort to understand the sentence is related to eventual recall of the sentence if effects due to repetition of information are controlled. Recall rates for the easy-appropriate and difficult-appropriate conditions are predicted to be equal, thus replicating the results obtained for the easy-postcue and difficult-postcue conditions in Experiment 1. It is further expected that comprehension will generally not be achieved in the difficult-inappropriate condition and that, consequently, recall rates will be substantially lower than in the other three conditions (cf. Auble \& Franks, 1978, Experiment 4).

\section{EXPERIMENT 2}

\section{Method}

Subjects. The subjects were 20 undergraduate volunteers at Vanderbilt University. They were either given credit in an introductory psychology course or paid for their participation in the experiment.

Materials and Procedure. A list of 24 sentences were used as stimuli. The first two and last two sentences in the list were filler items designed to reduce primacy and recency effects in the critical list. These items were not used in the analysis. Ten of the critical sentences were drawn from the pool of 20 easy sentences and 10 were drawn from the pool of 20 difficult sentences. Half of the easy sentences were presented with an appropriate cue $5 \mathrm{sec}$ after the sentence (easy-appropriate), and half were presented with an inappropriate cue $5 \mathrm{sec}$ after the sentence (easy-inappropriate). Similarly, half of the difficult sentences were presented with an appropriate cue after a 5-sec delay (difficult-appropriate) and half with an inappropriate cue (difficult-inappropriate). The inappropriate cues were chosen from the appropriate cues for the sentences in the pool that were not used in that particular acquisition list. The four types of sentences were randomly intermixed during acquisition. Four different acquisition lists were formed and counterbalanced such that each easy and difficult sentence appeared once with an appropriate cue and once with an inappropriate cue. The experimental procedure was identical to that in Experiment 1 except that only $8 \mathrm{~min}$ were given for free recall because of the shorter acquisition list.

\section{Results and Discussion}

The number of "understood" responses reported during acquisition was scored for the four conditions. As in Experiment 1, the conditions in which a sentence was followed by its appropriate cue appeared to be relatively well understood (easy-appropriate, mean $=98 \%$; difficult appropriate, mean $=81 \%$ ). Again, the easy sentences appeared to be comprehended slightly better than the more difficult sentences. Sentences in the difficultinappropriate condition were not well understood during acquisition (mean $=17 \%$ ), and sentences in the easy-inappropriate condition were reported as only moderately well understood (mean $=49 \%$ ). The latter result is probably due to a certain amount of confusion caused by the inappropriate cues. Some subjects reported that, although they had understood the easy sentence prior to the cue, receiving an inappropriate cue had led them to believe that they may not have understood the sentence after all.

Recall results were scored as in Experiment 1. Recall was highest in the difficult-appropriate and in the two easy conditions (difficult-appropriate, mean $=26 \%$, $\mathrm{SD}=18.5$; easy-appropriate, mean $=22 \%, \mathrm{SD}=19.6$; easy-inappropriate, mean $=24 \%, \mathrm{SD}=23.0$ ) and lowest in the difficult-inappropriate condition (mean $=10 \%$, $\mathrm{SD}=10.9$ ). The results were analyzed by a 4 by 2 by 2 mixed design analysis of variance with counterbalanced lists as the between-subjects factor and the easy-difficult and appropriate-inappropriate manipulations as withinsubjects factors. The interaction between the easydifficult and appropriate-inappropriate factors was significant $[F(1,57)=5.87, p<.025]$. The main effect for the appropriate-inappropriate factor was marginally significant $[F(1,57)=3.40, p<.08]$. No other main effects or interactions were significant. Comparison between the easy-appropriate and easy-inappropriate conditions revealed no difference in recall rates $[\mathrm{t}(57)=$ .41]. This indicates that the potential repetition of information provided when an appropriate cue followed an easy sentence did not enhance recall rates relative to the easy-inappropriate condition, in which no such repetition occurred. The difference between the difficultappropriate condition and the two easy conditions was also not significant $[t(57)=.66]$. (Obviously, the comparison between the easy-inappropriate and difficultappropriate conditions also was not significant $[t(57)=$ .37].) This result replicates the finding obtained in Experiment 1 for the easy-postcue and difficultpostcue conditions. In conjunction with the results of Experiment 1, this further supports the interpretation that an "aha" experience may be a more important factor in facilitating recall than the amount of elaborative processing involved. Comparison between the difficult-inappropriate condition and the remaining 
three conditions also provides tangential support for this position. Results were highly significant $[t(57)=3.23$, $\mathrm{p}<.01$ ], suggesting that recall is lower for cases such as the difficult-inappropriate condition, in which the initial noncomprehension of the sentence is not followed by the eventual understanding that is involved in an "aha" experience.

The low comprehension ratings for the easyinappropriate condition were not reflected in later recall rates. As noted above, only $49 \%$ of the sentences were reported "understood" during acquisition in this condition, while "understood" ratings for the difficult. appropriate and easy-appropriate conditions were $81 \%$ and $98 \%$, respectively. As discussed above, these ratings for the easy-inappropriate condition probably reflect confusion in the use of the rating scale when an inappropriate cue is presented, rather than noncomprehension of the sentences themselves. To examine whether the differential comprehension ratings in the three conditions were related to later recall, the recall results were rescored conditionally on subjects reporting the sentences "understood" during acquisition. If comprehension of the sentences in the easy-inappropriate condition was actually lower than in the difficultappropriate and easy-appropriate conditions, it would be expected that recall rates would rise for this condition when only sentences reported as "understood" were considered (cf. Auble \& Franks, 1978).

In contrast, if confusion caused by the presentation of an inappropriate cue was the main factor in the lower comprehension ratings for the easy-inappropriate condition, it would be expected that rescoring recall conditionally on "understood" responses would have little or no effect on recall rates for this condition. The latter expectation was confirmed by the rescoring. It was found that the conditional recall rates for the easyinappropriate condition were actually slightly lower than the unconditional recall rates (mean $=19 \%$ vs. mean $=$ $24 \%$ ). Recall rates were similarly unaffected for the difficult-appropriate and easy-appropriate conditions (difficult-appropriate, mean $=24 \%$; easy-appropriate, mean $=23 \%$ ). This supports the interpretation that sentences in the easy-inappropriate condition were relatively well understood and that presentation of the inappropriate cue caused subjects to be unsure of their interpretation of the sentence. Although the conditional recall data indicate that sentences in the easy-appropriate condition were recalled slightly better than sentences in the easy-inappropriate condition (as expected if effects due to the repetition of information were operating), this difference was not significant $[t(19)=.46]$. To check whether the differential comprehension ratings for easy and difficult sentences in Experiment 1 had any effect on later recall, results for this experiment were also rescored conditionally on whether subjects reported the sentences "understood" during acquisition. Once again, it was found that the pattern of results did not differ greatly from that in the unconditional scoring (easypostcue, mean $=29 \%$; difficult-postcue, mean $=34 \%$; easy-within, mean $=21 \%$; difficult-within, mean $=20 \%$ ).

Thus, the results of Experiments 1 and 2 suggest that, at least under the present experimental conditions, the "aha" process alone may be sufficient to promote recall. More specifically, recall of difficult sentences followed by appropriate cues was approximately equal to recall of easy sentences followed by either appropriate or inappropriate cues. However, a possible reinterpretation of these data still remains. It is possible that the presentation of a cue, any cue, following a sentence that can be understood in isolation (i.e., an "easy" sentence) might be seen as a cue evoking further retrieval of the sentence in the attempt to integrate the two. The cue-evoked reencoding of the sentence might then provide a second memory trace in addition to the original encoding of the sentence. This redundancy might facilitate recall of easy-postcue sentences. If this hypothesis holds, then recall rates should be lower for a condition in which easy sentences are not followed by a cue. Such a finding could indicate that elaboration effects are operative in all postcue conditions, but the hypothesized greater elaborative effects (and thus greater recall) for the difficult sentences may have been offset by redundant memory traces enhancing recall for the easy sentences. Note that one could argue that postcues for difficult sentences could also evoke such redundant reencodings of the sentence and thus not differ from easy sentences in this respect. In fact, this resembles one of the proposed possible mechanisms underlying the "aha" hypothesis mentioned in the introduction. However, a difference would remain between the encodings of the difficult and the easy sentences. The encoding of the easy sentence would involve comprehension of the sentence prior to the cue. The encoding of the difficult sentence would be a noncomprehended encoding. Much work, including results in Auble and Franks (1978), has demonstrated that comprehended encodings lead to much greater recall than do noncomprehended encodings. Thus, the potential recall enhancement due to redundancy in encoding easy sentences may be greater than any such effects with difficult sentences. And, thus, the obscuring of differences in elaboration effects discussed above remains a viable possibility. To examine this possibility, a third experiment using easy sentences was conducted, in which both postcues and the experience of an "aha" reaction were manipulated. Both the easy-appropriate and the easy-inappropriate conditions from Experiment 2 were again used, these being conditions that both receive a postcue and lead to "aha" reactions. Further, a condition was introduced in which easy sentences were presented without cues (easy/no-cue condition). In this condition, an "aha" experience should occur, but without a postcue that might lead to further retrieval and increased recall due to redundant encoding. The final condition used was the easy-within condition included in Experiment 1, in which the cue was meaningfully embedded in the sentence. In this condition, "aha" reactions would be minimized, and the presence of postcues that could 
facilitate elaboration and redundancy effects would be controlled. If the "aha" experience is indeed the most important determinant of later recall rates, then the easy-appropriate, easy-inappropriate, and easy/no-cue conditions should be equally well recalled, and all should be recalled better than the easy-within condition, in which the occurrence of such experiences is minimized. On the other hand, if the presence of a postcue does lead to reencoding of the sentence and thus enhances subsequent recall, it would be predicted that recall in the easy-appropriate and easy-inappropriate conditions would be higher than in the easy/no-cue condition. As a replication of Experiment 2, equal recall rates are expected for the easy-appropriate and easy-inappropriate conditions.

\section{EXPERIMENT 3}

\begin{abstract}
Method
Subjects. The subjects were 20 undergraduate volunteers at Vanderbilt University. They were either given credit in an introductory psychology course or paid for their participation in the experiment.

Materials and Procedure. A list of 24 easy sentences was used, with the first two and last two sentences serving as filler items. Sentences were presented (1) with an appropriate cue following the sentence after a 5-sec delay (easy-appropriate), (2) with an inappropriate cue following the sentence after a $5-\mathrm{sec}$ delay (easy-inappropriate), (3) with the appropriate cue meaningfully embedded in the sentence (easy-within), or (4) with no cue (easy/no-cue). A 5-sec delay occurred after each sentence for the easy-within and easy/no-cue conditions. The four types of sentences were randomly intermixed during acquisition. Four different acquisition lists were formed and counterbalanced such that each sentence appeared in all four conditions over lists. The experimental procedure was identical to that of Experiment 2 .
\end{abstract}

\section{Results and Discussion}

Results were scored as in Experiments 1 and 2. Scoring the comprehension ratings obtained during acquisition indicated that the conditions receiving appropriate cues, no cues, or the cues embedded in the sentences were relatively well understood (easyappropriate, mean $=92 \%$; easy $/$ no-cue, mean $=84 \%$; easy-within, mean $=86 \%$ ). The condition receiving inappropriate cues was reported as only moderately well understood (easy-inappropriate, mean $=24 \%$ ). As discussed in Experiment 2, the latter finding is probably due to confusion that arises when an inappropriate cue is presented. Recall results indicated that recall rates for the conditions receiving appropriate cues, inappropriate cues, or no cues were generally higher than for the condition in which the cues were embedded in the sentences (easy-appropriate, mean $=29 \% ; \quad \mathrm{SD}=17.7$; easyinappropriate, mean $=24 \%, \mathrm{SD}=19.0$; easy $/$ no-cue , mean $=23 \%, \quad \mathrm{SD}=19.8 ; \quad$ easy-within, mean $=15 \%$, $\mathrm{SD}=17.0$ ). As in Experiments 1 and 2, the data were also scored conditionally on reported comprehension of the sentence during acquisition. Again, the pattern of results remained essentially the same as the pattern obtained from nonconditional scoring (easy-appropriate, mean $=30 \%$; easy-inappropriate, mean $=29 \%$; easy $/$ nocue, mean $=24 \%$; easy-within, mean $=13 \%$ ). The results of the nonconditional scoring were used in further analysis of the data.

The results were analyzed by a 4 by 4 mixed design analysis of variance with the four counterbalanced lists as the between-subjects factor and the four types of sentences as the within-subjects factor. The main effect due to sentence type was marginally significant $[F(1,57)=2.09, p=.10]$. No other main effects or interactions were significant. Following Kirk (1968), the specific a priori comparisons warranted by the hypotheses leading to the experiment were examined. Comparison between the easy-appropriate and easyinappropriate conditions revealed no significant difference in recall rates $[t(57)=.88]$. This replicates the finding of Experiment 2 and again suggests that the type of cue does not affect recall rates when the sentence can be comprehended in isolation. To examine whether potential redundancy effects induced by postcuing were operative in addition to elaborative processes, the recall results for the easy-appropriate and easyinappropriate conditions were compared with the results for the easy/no-cue condition. Results were not significant $[\mathrm{t}(57)=.71]$, suggesting that any redundancy of encodings induced by the presence of a postcue (as in the easy-appropriate and easy-inappropriate conditions) was not an effective variable in promoting recall relative to the easy/no-cue condition, in which any such cueinduced redundancy effects were controlled. In other words, whether the cue is appropriate or inappropriate to the sentence, or even whether the cue is given at all, does not seem to affect recall rates as long as an initial noncomprehension of the sentence is followed by eventual understanding (i.e., an "aha" experience occurs).

A significant difference was obtained when the three conditions involving "aha" reactions (easy-appropriate, easy-inappropriate, easy/no-cue) were compared with the easy-within condition, in which no such reactions were present $[t(57)=2.23, p<.05]$. This result upholds the conclusion drawn in Experiments 1 and 2 that an "aha" experience is importantly related to later recall. Conditions in which subjects experience an "aha" reaction exhibit facilitated recall rates when compared with conditions in which the initial noncomprehension of the sentence is not present ("within" conditions). Results from Experiments 2 and 3 also indicate that potential cue-induced redundancy effects did not artifactually enhance recall in the easy-appropriate condition relative to the difficult-appropriate postcue conditions in Experiments 1 and 2.

Thus, the results of the first three experiments suggest that the amount of elaboration involved in comprehending a sentence may not be an important determinant of later recall rates under these conditions. Instead recall seems to be facilitated in conditions in which "aha" reactions are experienced. This finding suggests that the results of Auble and Franks (1978, Experi- 
ment 1) demonstrating facilitated recall when extra time is given for conditions in which the cue is presented after the sentence can now be reinterpreted in terms of an "aha" experience requiring a certain amount of time to occur. Further, the discovery that extra time had no effect on recall for conditions in which the cue was meaningfully embedded in the sentence (Auble \& Franks, Experiment 3) can now be viewed in terms of the absence of an "aha" experience in these conditions and a consequential lack of effectiveness of the extra time given.

However, what of cases such as the difficultinappropriate condition or the difficult/no-cue conditions (used by Auble \& Franks, 1978, Experiment 4), in which an "aha" reaction does not occur because the sentence remains incomprehensible? Is the elaborative processing that presumably occurs in the attempt to understand the sentences an effective determinant of later recall in these cases? Auble and Franks (Experiment 4) found that in conditions in which no cue was presented after a difficult sentence, extra time following the sentence had no effect on later recall. From this result, it was concluded that more time may be ineffective when comprehension (and an "aha" experience) is not ultimately achieved. In other words, elaborative processing that may occur in the longer time intervals is ineffective when the sentence remains incomprehensible. However, an alternative interpretation of this result is also available. It is possible that, when difficult sentences without cues are used as materials, subjects may assume that solving this type of sentence is impossible and thus not be motivated to attempt to comprehend it, even when extra time is given to do so. In contrast, if subjects were given an inappropriate cue following the sentence, they might at least attempt to elaboratively integrate the cue and the sentence. Even if unsuccessful, this elaborative effort may enhance later recall rates relative to a no-cue condition. Although no evidence was found supporting such elaboration effects in the previously discussed postcue conditions, all of these conditions involved "aha" reactions. Thus, it is still possible that elaborative effects could influence recall in the absence of "aha" experiences. The fourth experiment was designed to examine this hypothesis by using both difficult sentences with no cues (difficult/no-cue condition) and difficult sentences followed by inappropriate cues (difficult-inappropriate condition).

A second purpose of Experiment 4 was to extend the findings of Auble and Franks (1978) by directly comparing recall rates for the two conditions that do not produce "aha" reactions. Note that the lack of "aha" experiences in the difficult/no-cue and within conditions occur for different reasons. In the first case, the initial noncomprehension of the sentence is generally not followed by understanding (i.e., no "aha" experience occurs). In the within conditions, an "aha" experience fails to occur because the sentences are generally understood immediately upon presentation. Thus, there is no initial noncomprehension followed by comprehension (i.e., no "aha" experience). Although Auble and Franks found that recall for both of these conditions was lower than that for conditions that do produce "aha" reactions, the difficult/no-cue and difficult-within conditions were never directly compared. It is possible, and indeed even likely, that factors such as comprehensibility of the sentences are operating and may result in differential recall rates for these two conditions. To examine this possibility, both the difficult-within and the difficultappropriate (which does produce "aha" reactions) conditions were also included in Experiment 4.

\section{EXPERIMENT 4}

\section{Method}

Subjects. The subjects were 20 undergraduate volunteers at Vanderbilt University. They were either given credit in an introductory psychology course or paid for their participation in the experiment.

Materials and Procedure. The acquisition list was constructed in the same manner as in Experiment 3, except that difficult sentences were used instead of easy sentences. There were four experimental conditions: (1) The sentence was followed by an appropriate cue (difficult-appropriate); (2) the cue was meaningfully embedded in the sentence (difficult-within); (3) the sentence was followed by an inappropriate cue (difficult-inappropriate) and (4) the sentence was presented alone (difficult/no-cue). For the difficult-appropriate and difficult-inappropriate conditions, a 5 -sec delay occurred between the sentence and the cue. The 5-sec delay occurred after the sentence for the difficult/ no-cue and difficult-within conditions. The experimental procedure was identical to Experiments 2 and 3.

\section{Results and Discussion}

Comprehension ratings obtained during acquisition indicated that sentences in the difficult-appropriate and difficult-within conditions were relatively well understood (difficult-appropriate, mean $=82 \%$; difficultwithin, mean $=82 \%$, while sentences in the difficultinappropriate and difficult/no-cue conditions were rarely comprehended (difficult-inappropriate, mean = $11 \%$; difficult $/$ no-cue, mean $=15 \%$ ). Recall rates were scored as in the previous experiments. Recall was highest in the difficult-appropriate condition (mean = $34 \%, \mathrm{SD}=25.2$ ), lower in the difficult-within condition (mean $=22 \%, \mathrm{SD}=19.4$ ), and relatively poor in the difficult/no-cue and difficult-inappropriate conditions (difficult/no-cue, mean $=12 \%, \quad S D=17.6$; difficultinappropriate, mean $=9 \%, \mathrm{SD}=13.7$ ).

Results were analyzed by a 4 by 4 mixed design analysis of variance with the counterbalanced lists as the between-subjects factor and the types of sentences as the within-subjects factor. The only significant effect was the main effect for sentence type $[F(3,57)=7.72$, $p<.001]$. Comparing the difficult-inappropriate and difficult/no-cue conditions revealed no significant difference in recall rates $[\mathrm{t}(57)=.52]$. This finding does not support the notion that the low recall rates in the difficult/no-cue condition may be partially due to subjects' refusal to even attempt to elaborate and try to understand the sentences in this condition. If this potential lack of motivation had been influential, then 
recall in the difficult-inappropriate condition (in which subjects presumably attempt to integrate the sentence and cue) might be expected to have been enhanced relative to the difficult/no-cue condition. This reinforces the conclusions drawn previously that elaborative processing may be ineffective for later recall under the present conditions, and, more specifically, it provides support for the notion that elaboration may not be useful when comprehension is not ultimately achieved.

A significant difference was obtained when recall for the difficult-within condition was compared with recall in the difficult-inappropriate and difficult/no-cue conditions $[\mathrm{t}(57)=2.31, \mathrm{p}<.05]$. This result indicates that, even without an "aha" experience, successful comprehension of the sentences (as in the within condition) enhances recall relative to conditions such as the difficult-inappropriate and difficult/no-cue conditions, in which comprehension is generally not achieved.

To examine whether "aha" experiences facilitated recall as in Experiments 1-3, recall for the difficultappropriate condition was compared with the remaining conditions. Comparisons were made with the difficultwithin condition alone and with the three remaining conditions pooled. Results for both comparisons were significant $[t(57)=2.09, p<.025$, and $t(57)=4.19$, $\mathrm{p}<.01$, respectively]. Once again, this substantiates the importance of an "aha" experience in promoting recall. It seems that the initial noncomprehension of the sentences followed by understanding in the difficultappropriate condition is influential in enhancing recall rates when compared with conditions in which "aha" experiences do not occur. Elimination of the "aha" experience, either by providing an immediately compre. hensible sentence (the within conditions) or because the initial noncomprehension of the sentence is never resolved into eventual understanding (as in the difficult/ no-cue or difficult-inappropriate conditions) seems to result in a deficit in recall relative to conditions in which the "aha" response is present.

\section{CONCLUSION}

The present experiments have demonstrated that initial noncomprehension of a sentence followed by comprehension (an "aha" experience) facilitates recall relative both to conditions in which this initial comprehension does not occur and to conditions in which the sentence is not ultimately understood. Further, the evidence from these studies indicates that the amount of elaborative processing involved in understanding a sentence does not seem to be an effective determinant of later recall. More specifically, equal recall rates were obtained for relatively difficult-to-comprehend sentences and easy-to-comprehend sentences. Control conditions indicate that the equal recall rates in these conditions were not due to the implicit repetition of information occurring when an appropriate cue was presented after an easy sentence. Recall rates for the relatively easyto-comprehend sentences also remained the same when the sentences were presented without cues, suggesting that further elaborative processes that could be evoked when a postcue is presented were not effective in facilitating recall of the easy sentences. Further, it was found that potential elaborative processes contingent upon a postcue were ineffective in enhancing recall of difficult sentences when those sentences remained incomprehensible.

Finally, although this work supports the effectiveness of the "aha" process in enhancing recall, the precise mechanisms that underlie these effects remain speculative. In the introduction, two processes were considered as possible bases for the "aha" effect. It was suggested that the "aha" process might be seen as a sequence of two encoded representations. The first is a noncomprehended representation of the sentence without contextual reference, and the second, a comprehended representation of the sentence integrated with the contextual reference as expressed in the appropriate cue. One suggested possible basis for enhanced recall under "aha" conditions was that the two representations lead to a repetition or redundancy of stored information, and this redundancy of encoded representations enhanced retrieval. Although some forms of this interpretation may still be viable, the lack of evidence for effects attributable to redundancy in Experiments 2 and 3 make this account of the "aha" effect less plausible. A second possible interpretation would be to attribute the effect to the normative distinctiveness of the "aha" reaction itself. It was suggested that, compared with everyday language processing, in which sentences are usually readily understood, the state of noncomprehension followed by comprehension induced by the sentences used in the present work is a highly distinctive psychological state. This distinctiveness may be represented in the encoding of the sentences and may form a relatively efficient retrieval route.

A third possible interpretation would be to attribute the enhanced recall in conditions experiencing "aha" reactions to integration processes involved in transforming the initial noncomprehended representation of the sentence into the second comprehended representation. One might characterize this as a situation in which the subjects have partial information (provided by the separate sentences and cues) and must retrieve or generate a representation that meets the requirements of the acquisition task (i.e., to comprehend the sentences). This process may be psychologically similar to the situation of the subjects at time of recall in which they again may have partial information and must attempt to retrieve or generate a representation that meets the task requirements (i.e., recall the sentences). It may be that, in this sense, the recall test conditions are more similar to the postcue than to the within-cue acquisition conditions. If this is the case, then enhanced recall for postcue conditions may be attributed to a type of encoding specificity principle (Tulving \& Thomson, 1973) or to transfer-appropriate processing (Bransford, Franks, Morris, \& Stein, 1979), in which similarity of acquisition 
and test conditions enhances test performance. Further research is necessary to determine the relative adequacy of these interpretations of the "aha" effect.

\section{REFERENCES}

Auble, P. M., \& Franks, J. J. The effects of effort toward comprehension on recall. Memory \& Cognition, 1978, 6, 20-25.

Bransford, J. D., Franks, J. J., Morris, C. D., \& Stein, B. S. Some general constraints on learning and memory research. In L. S. Cermak \& F. I. M. Craik (Eds.), Levels of processing and human memory. Hillsdale, N.J: Erlbaum, 1979.

Bransford, J. D., \& McCarrell, N. S. A sketch of a cognitive approach to comprehension: Some thoughts about understanding what it means to comprehend. In W. B. Weimer \& D. Palermo (Eds.), Cognition and the symbolic processes. Hillsdale, N.J: Erlbaum, 1974.

CLARK, H. H. The language-as-fixed-effect fallacy: A critique of language statistics in psychological research. Journal of Verbal
Learning and Verbal Behavior, 1973, 12, 335-359.

Craik, F. I. M., \& Lockhart, R. S. Levels of processing: A framework for memory research. Journal of Verbal Learning and Verbal Behavior, 1972, 11, 671-684.

Craik, F. I. M., \& Tulving, E. Depth of processing and the retention of words in episodic memory. Journal of Experimental Psychology: General, 1975, 104, 268-294.

Forster, K. I., \& Dickinson, R. G. More on the language-asfixed-effect fallacy: Monte Carlo estimates of error rates for $F_{1}, F_{2}, F^{\prime}$, and min $F^{\prime}$. Journal of Verbal Learning and Verbal Behavior, 1976, 15, 135-142.

Johnson, M. K., Dole, T. J., Bransford, J. D., \& Lapinski, R. H. Context effects in sentence memory. Journal of Experimental Psychology, 1974, 103, 358-360.

KIRK, R. E. Experimental design: Procedures for the behavioral sciences. Belmont, Calif: Brooks-Cole, 1968.

Tulving, E., \& Thomson, D. M. Encoding specificity and retrieval processes in episodic memory. Psychological Review, $1973,80,352-373$.

Appendix A

Easy and Difficult Sentences with Appropriate Cues

\begin{tabular}{|c|c|c|c|}
\hline Percent* & & & Cue \\
\hline \multicolumn{4}{|c|}{ Easy Sentences } \\
\hline 100 & 1. & The office was cool because the windows were closed. & air conditioned \\
\hline 100 & 2. & The man got well because the slicing was successful. & operation \\
\hline 100 & 3. & The coffee was sweet because the powder was inserted. & sugar \\
\hline 95 & 4. & The Arab was sad because the humps shrunk. & thirsty camel \\
\hline 95 & 5. & The engine stopped because the liquid ran out. & gasoline \\
\hline 95 & 6. & The house tumbled because the insects were satisfied. & termites \\
\hline 95 & 7. & The lady lost the gold because the dentist jerked hard. & pulling a tooth \\
\hline 95 & 8. & The colors appeared because the rain stopped. & rainbow \\
\hline 95 & 9. & Her toes were pointed because the dance started. & ballet \\
\hline 95 & 10. & The teacher stopped writing because the stick crumbled. & chalk \\
\hline 89 & 11. & The car was moved because he had no change. & parking meter \\
\hline 89 & 12. & The pioneers were safe because the circle was completed. & covered wagon \\
\hline 84 & 13. & The antelope ran because the spots shifted. & leopard \\
\hline 84 & 14. & The numbers were added because the button was pushed. & calculator \\
\hline 84 & 15. & The criminal fled because the red bubble flashed. & police car \\
\hline 79 & 16. & The airplane changed altitude because the force was strong. & high wind \\
\hline 79 & 17. & The bicycle was useful because the air was increased. & flat tire \\
\hline 74 & 18. & The child turned because her pet talked. & parrot \\
\hline 74 & 19. & The betting was halted because the wheel was spun. & roulette \\
\hline 63 & 20. & The boy's chest hurt because his friend pulled the straps. & suspenders \\
\hline \multicolumn{4}{|c|}{ Difficult Sentences } \\
\hline 0 & 1. & The food was intact because the plate fell apart. & dentures \\
\hline 0 & 2. & The party was stalled because the wire straightened. & corkscrew \\
\hline 0 & 3. & The girl spilled her popcorn because the lock broke. & lion cage \\
\hline 0 & 4. & The needle fell because the bucket was not filled. & christmas tree \\
\hline 0 & 5. & The person was unhappy because the hole closed. & pierced ears \\
\hline 0 & 6. & The breakfast was excellent because the thread was sticky. & spiderweb \\
\hline 5 & 7. & The home was small because the sun came out. & igloo \\
\hline 5 & 8. & The street was full of holes because the turning stopped. & cement mixer \\
\hline 5 & 9. & The clothes were ruined because the sign vanished. & wet paint \\
\hline 10 & 10. & The smell began because the signs went up. & garbage strike \\
\hline 11 & 11. & The dinner was uneaten because the wood was warped. & chopsticks \\
\hline 16 & 12. & The haystack was important because the cloth ripped. & parachute \\
\hline 16 & 13. & The woman could not see because the blade was bent. & windshield wiper \\
\hline 21 & 14. & The audience cheered because the five balls rotated. & juggler \\
\hline 21 & 15. & The family asked for rooms because the light had burned out. & no vacancy \\
\hline 21 & 16. & The cut was burned because the clock stopped. & cooking meat \\
\hline 21 & 17. & The notes were sour because the seam split. & bagpipe \\
\hline 21 & 18. & The tree grew because the wrinkles opened. & peach pit \\
\hline 26 & 19. & The man's back ached because the ends were too large. & barbells \\
\hline 37 & 20. & The boy was hungry because the pole splintered. & fishing \\
\hline
\end{tabular}

*Percentage of subjects who rated each sentence as understandable without the appropriate cue.

(Received for publication March 14, 1979; revision accepted August 25, 1979.) 